University of Nebraska - Lincoln

DigitalCommons@University of Nebraska - Lincoln

Faculty Publications from the Department of

Electrical and Computer Engineering

2-14-2002

\title{
Nanostructures with small- and large-scale periodic and random rough surfaces and lateral fluctuations in the permittivity and permeability-scattered near fields
}

Ezekiel Bahar

Follow this and additional works at: https://digitalcommons.unl.edu/electricalengineeringfacpub

Part of the Computer Engineering Commons, and the Electrical and Computer Engineering Commons

This Article is brought to you for free and open access by the Electrical \& Computer Engineering, Department of at DigitalCommons@University of Nebraska - Lincoln. It has been accepted for inclusion in Faculty Publications from the Department of Electrical and Computer Engineering by an authorized administrator of DigitalCommons@University of Nebraska - Lincoln. 


\section{Nanostructures with small- and large-scale periodic and random rough surfaces and lateral fluctuations in the permittivity and permeability-scattered near fields}

\author{
Ezekiel Bahar \\ University of Nebraska-Lincoln \\ Electrical Engineering Department and the \\ Center for Electro-Optics \\ Lincoln, Nebraska 68588-0511 \\ E-mail: ebahar1@unl.edu
}

\begin{abstract}
The scattered electromagnetic near fields due to fluctuations in the surface height and/or lateral variations in the electromagnetic medium parameters are evaluated using a full-wave approach. Since the scales of the height and medium fluctuations considered could be significantly smaller or larger than the electromagnetic wavelengths, the familiar perturbation and physical/geometrical optics solutions cannot be used nor is it possible to investigate subwavelength features based on far-field measurements. The full-wave approach employs complete field expansion that includes propagating and evanescent waves as well as lateral waves and surface waves, which are not accounted for in the perturbation and physical optics solutions. Exact boundary conditions for the electric and magnetic fields are imposed at the rough interfaces. The complete modal expansions do not uniformly converge at the irregular boundaries, therefore the interchange of orders of differentiation and summation/integration of the field expansions is avoided. Large-scale and small-scale (relative to electromagnetic wavelength) approximations for the full-wave near-field solutions are expressed as integrals over the complete scattered wave number variables. Random rough surfaces as well as periodic and quasiperiodic structures are also considered. (c) 2002 Society of Photo-Optical Instrumentation Engineers. [DOI: 10.1117/1.1489050]
\end{abstract}

Subject terms: near fields; electromagnetic wave scattering; lateral waves; surface waves; irregular stratified media; nanostructures.

Paper 010362 received Oct. 6, 2001; revised manuscript received Feb. 8, 2002; accepted for publication Feb. 14, 2002.

\section{Introduction}

Using the full-wave approach summarized here, complete expansions for the electric and magnetic fields are employed. These include the radiation fields, the lateral waves, and the surface waves of the structure. Thus, the electromagnetic fields are expressed ${ }^{1-3}$ completely in terms of infinite integrals (associated with two branch cuts) as well as guided waves (associated with residues). Since the familiar Fresnell reflection and transmission coefficients or the impedance boundary condition do not adequately represent the fields at the interfaces, exact boundary conditions are imposed at each of the interfaces of the medium of propagation. Maxwells equations are converted into sets of firstorder coupled differential equations (referred to as generalized telegraphists' equations) for the scattered wave amplitudes. The coupling between the wave amplitudes is due to the lateral variations of the height of the interfaces in the stratified media and the lateral flucuations in the complex permittivities and permeabilities of the media. Largescale and small-scale (relative to the electromagnetic wavelength) approximations to the full-wave near-field solutions are expressed as double integrals over the scattered wave vector variables. Random rough surfaces as well as periodic and quasiperiodic structures are also considered in this work. The impact of surface roughness and medium fluc- tuations on the optical characterization of stratified structures can be determined. Much of the current work on nearfield scattering from rough surfaces is based on numerical approaches.

This work is applicable to the synthesis and modification of nanostructures and contributes to the fundamental understanding of the physical characteristics of artificially engineered stratified structures with nanoscale roughness and lateral fluctuations of its electrical and magnetic properties.

\section{Formulation of the Generalized Telegraphists' Equation}

The full-wave procedures outlined here are used to convert Maxwell's equations into generalized telegraphists' equations for propagation of electromagnetic waves in irregular layered structures with 2-D rough interfaces and laterally varying electromagnetic parameters. ${ }^{1-3}$ The vertical axis is $y$ and the interface between medium $i$ and $i+1$ is given by $f(x, y, z)=y-h_{i, i+1}(x, z)=0$. The complex permittivity $\varepsilon$ and the permeability $\mu$ in each layer of the structure are assumed to be functions of the lateral variables $x$ and $z$.

Maxwell's equation for the transverse $(y, z)$ components (denoted by subscript $T$ ) of the electric $\bar{E}$ and magnetic $\bar{H}$ fields can be expressed as follows: 


$$
\begin{aligned}
-\frac{\partial \bar{E}_{T}}{\partial x}= & i \omega \mu\left(\bar{H}_{T} \times \bar{a}_{x}\right)-\frac{1}{i \omega} \nabla_{T}\left[\frac{1}{\varepsilon} \nabla_{T} \cdot\left(\bar{H}_{T} \times \bar{a}_{x}\right)\right] \\
& +\bar{M}_{T} \bar{a}_{x}+\frac{1}{i \omega} \nabla_{T} \frac{J_{x}}{\varepsilon}
\end{aligned}
$$

and

$$
\begin{aligned}
-\frac{\partial \bar{H}_{T}}{\partial x}= & i \omega \varepsilon\left(\bar{a}_{x} \times \bar{E}_{T}\right)-\frac{1}{i \omega} \nabla_{T}\left[\frac{1}{\mu} \nabla_{T} \cdot\left(\bar{a}_{x} \times \bar{E}_{T}\right)\right]+\bar{a}_{x} \times \bar{J}_{T} \\
& +\frac{1}{i \omega} \nabla_{T} \frac{M_{x}}{\mu}
\end{aligned}
$$

in which the operator $\nabla_{T}$ is

$$
\nabla_{T}=\bar{a}_{y} \frac{\partial}{\partial y}+\bar{a}_{z} \frac{\partial}{\partial z}
$$

and the transverse vectors are

$\bar{A}_{T}=\bar{a}_{y} A_{y}+\bar{a}_{z} A_{z}$.

The electric and (dual) magnetic current densities are $\bar{J}\left(A / \mathrm{m}^{2}\right)$ and $\bar{M}\left(\mathrm{~V} / \mathrm{m}^{2}\right)$. The exact boundary conditions imposed at each of the interfaces of the irregular layered structure are the continuity of the tangential components of the electric and magnetic fields

$(\bar{n} \times \bar{E})_{h_{i-1, i}^{-}}^{h_{i-1, i}^{+}}=0,(\bar{n} \times \bar{H})_{h_{i-1, i}^{-}}^{h_{i, i}^{+}}=0, \quad i=1, \ldots, m$,

in which $\bar{n}$ is the unit vector normal to the interfaces

$$
\begin{aligned}
\bar{n}_{i, i+1}= & \left(-\frac{\partial h_{i, i+1}}{\partial x}, 1,-\frac{\partial h_{i, i+1}}{\partial z}\right) /\left[\left(\frac{\partial h_{i, i+1}}{\partial x}\right)^{2}+1\right. \\
& \left.+\left(\frac{\partial h_{i, i+1}}{\partial z}\right)^{2}\right]^{1 / 2}
\end{aligned}
$$

and $y=h_{i-1, i}$ is the interface between medium $i-1$ and medium $i$. The full-wave, complete expansions for the vertically $(V)$ and the horizontally $(H)$ polarized electric and magnetic fields are given in terms of the transverse basis functions $\bar{e}_{T}^{V}, \bar{h}_{T}^{V}, \bar{e}_{T}^{H}$, and $\bar{h}_{T}^{H}$.

The transverse components of the electric and magnetic fields are expressed completely as follows:

$\bar{E}_{T}(x, y, z)=\sum_{v} \int_{-\infty}^{\infty}\left[E^{V}(x, v, w) \bar{e}_{T}^{V}+E^{H}(x, v, w) e_{T}^{H}\right] \mathrm{d} w$,

and

$\bar{H}_{T}(x, y, z)=\sum_{v} \int_{-\infty}^{\infty}\left[H^{V}(x, v, w) \bar{h}_{T}^{V}+H^{H}(x, v, w) \bar{h}_{T}^{H}\right] \mathrm{d} w$, in which the symbol $\Sigma_{v}$ denotes summation (integration) over the complete wave vector spectrum consisting of the radiation term and lateral waves (associated with branch cut integrals) and the waveguide modes (or surface waves) of the layered structure (associated with the residues at the poles of the reflection coefficients) (Refs. 1-3,5,6). In Eqs. (7) and (8), the scalar field transforms for the vertically $(P=V)$ and horizontally $(P=H)$ polarized electric and magnetic fields are

$E^{P}(x, v, w)=\int_{-\infty}^{\infty} \bar{E}_{T}(x, y, z) \cdot\left(\bar{h}_{P}^{T} \times \bar{a}_{x}\right) \mathrm{d} y \mathrm{~d} z$,

and

$H^{P}(x, v, w)=\int_{-\infty}^{\infty} \bar{H}_{T}(x, y, z) \cdot\left(\bar{a}_{x} \times \bar{e}_{P}^{T}\right) \mathrm{d} y \mathrm{~d} z$,

where the complementary (reciprocal) basis functions are $\bar{e}_{V}^{T}, \bar{h}_{V}^{T}, \bar{e}_{H}^{T}$, and $\bar{H}_{H}^{T}$.

The basis functions and the reciprocal basis functions satisfy the biorthogonal relationships

$\left.\begin{array}{l}\int_{-\infty}^{\infty} \bar{e}_{T}^{P} \cdot\left(\bar{h}_{Q}^{T} \times \bar{a}_{x}\right)^{\prime} \mathrm{d} y \mathrm{~d} z \\ \int_{-\infty}^{\infty} \bar{h}_{T}^{P} \cdot\left(\bar{a}_{x} \times \bar{e}_{P}^{T}\right)^{\prime} \mathrm{d} y \mathrm{~d} z\end{array}\right\}=\delta_{P, Q} \Delta\left(v-v^{\prime}\right) \delta\left(w-w^{\prime}\right)$.

In Eq. (11) the Kronecker delta $\delta_{P, Q}$ implies that the vertically $(P, Q=V)$ and horizontally $(P, Q=H)$ polarized basis functions are orthogonal in uniform layered structures. Furthermore, the Dirac delta function $\delta\left(w-w^{\prime}\right)$ appearing in Eq. (11) is a result of the completeness and orthogonality relationships associated with the $z$ dependent functions.

The symbol $\Delta\left(v, v^{\prime}\right)$ in Eq. (11) is the product of the Kronecker delta $\delta_{q, r}$ (subscripts $q$ and $r$ denote the radiation fields, the later waves, or the surface waves) and the Dirac delta function $\delta\left(v, v^{\prime}\right)$ for the radiation and lateral wave terms or the Kronecker delta $\delta_{v, v^{\prime}}$ for the guided (surface) waves of the layered structure. Thus the radiation fields, the lateral waves, and the guided waves of the fullwave spectrum are mutually orthogonal. ${ }^{1,2}$ The radiation fields and the lateral waves are associated with branch cut integrals in the complex wave number plane (with branch points at $k=k_{0}$, uppermost medium and $k=k_{m}$, lowermost medium). The guided waves of the layered structure are associated with the residues at the poles of the composite reflection coefficient seen from above or below the layered structure.

In this paper, it is convenient to express the vertically and horizontally polarized scalar field transforms of Eqs. (9) and (10) in terms of the vertically and horizontally polarized forward wave amplitude $a^{P}$ and backward wave amplitude $b^{P}$ as follows: 
$H^{P}=a^{P} \pm b^{P}$ and $E^{P}=a^{P} \mp b^{P}$ for $P\left\{\begin{array}{l}V(\text { upper sign }) \\ H(\text { lower sign })\end{array}\right.$

Upon substituting the complete field transforms for the transverse components into Maxwell's equation [Eqs. (1)(4)], making use of the biorthogonal relationships of Eq. (11), and imposing the exact boundary conditions at each interface of the irregular layered structure of Eq. (5), the following generalized telegraphists' equations are derived:

$$
-\frac{\mathrm{d} a^{P}}{\mathrm{~d} x}-i u a^{P}=\sum_{Q} \sum_{v^{\prime}} \int_{-\infty}^{\infty}\left(s_{P Q}^{B A} a^{Q}+S_{P Q}^{B B} b^{Q}\right) \mathrm{d} w^{\prime}-A^{P},
$$

$$
-\frac{\mathrm{d} b^{P}}{\mathrm{~d} x}+i u b^{P}=\sum_{Q} \sum_{v^{\prime}} \int_{-\infty}^{\infty}\left(s_{P Q}^{A A} a^{Q}+S_{P Q}^{A B} b^{Q}\right) \mathrm{d} w^{\prime}+B^{P},
$$

in which $A^{P}$ and $B^{P}$ are associated with the source terms $\bar{J}$ and $\bar{M}$ in Eqs. (1)-(4). Furthermore, $S_{P Q}^{B A}$ and $S_{P Q}^{A B}$ are transmission scattering coefficients, while $S_{P Q}^{A A}$ and $S_{P Q}^{B B}$ are reflection scattering coefficients. These scattering coefficients vanish when the layered medium is horizontally stratified with homogeneous medium in each layer. In this case, the forward and backward wave amplitudes for the vertically and horizontally polarized waves are decoupled and analytical closed-form solutions are readily obtained. However, if the rough surface height or the complex permittivities and permeabilities are functions of $x$ and $z$, the lateral (horizontal) variables, the wave amplitudes are coupled. In the general case, the basis functions do not individually satisfy the irregular boundary conditions and the complete field expansions do not uniformally converge at the boundaries. Thus, on following precise mathematical procedures ${ }^{1-6}$ the orders of integration (summation) and differentiation are not interchanged.

The intrinsic properties of the full-wave solutions are duality, reciprocity, realizability, and invariance to coordinate transformations. All these properties follow directly from Maxwell's equations [Eqs. (1)-(4)] and they are not a result of any additional constraints imposed on the results. A 2-D scalarized version of this problem has also been analyzed. ${ }^{5,6}$ When the lowermost and/or uppermost half space is perfectly conducting or a good conducting medium, the two boundary conditions [Eqs. (5)] at the lowermost (and/or uppermost) interface can be replaced by a single surface impedance boundary condition.

$\bar{n} \times \bar{E}=Z_{s} \bar{n} \times \bar{H} \times \bar{n}$.

In Eq. (15) the unit vector $\bar{n}$ is normal to the interface and points into the conducting half space. For the isotropic conducting half space, the surface impedance $Z_{s}$ is a scalar. In general, the surface impedance can be represented by a dyad. The impedance boundary condition is often used to simplify the analysis of irregular layered structures. ${ }^{7}$ Contributions from integrals associated with one (or two) branch cuts are eliminated when impedance boundary con- ditions are used. The generalized telegraphists' equations have also been derived from irregular multilayered cylindrical structures. ${ }^{8,9}$

The scattering coefficients $S_{P Q}^{\alpha \beta}$ appearing in the generalized telegraphists' equations [Eqs. (13) and (14)] can be expressed in closed-form on employing the orthogonal relationships between the basis function. ${ }^{10}$ For example, if the rough surface height parameters $h$ and medium parameters $\varepsilon$ and $\mu$ are functions of $x$ only, the basis functions associated with the vertical $(y)$ variable are also implicitly dependent on the $x$ variable through the functions $h_{r-1, r}(x)$ (denoting the interface between medium $r-1$ and medium $r, r=1, \ldots, m)$ and the complex permittivities $\varepsilon_{r}(x)$ and permeabilities $\mu_{r}(x)$ in medium denoted by the subscript $r$.

The coupling coefficients can be expressed explicitly in terms of the derivatives of the scalar basis functions $\left[\partial \Psi_{p}(u, y)\right] / \partial x$ and $\left[\partial^{2} \Psi_{p}(u, y)\right] /(\partial x \partial y)$. Thus, in this case $\partial \Psi_{p} / \partial x$ can be expressed as

$$
\begin{aligned}
\frac{\partial}{\partial x} \Psi_{p}(u, y)= & {\left[\sum_{r=0}^{m}\left(\varepsilon_{r}^{\prime} \frac{\partial}{\partial \varepsilon_{r}}+\mu_{r}^{\prime} \frac{\partial}{\partial \mu_{r}}\right)\right.} \\
& \left.+\sum_{r=1}^{m} h_{r-1, r}^{\prime} \frac{\mathrm{d}}{\mathrm{d} h_{r-1, r}}\right] \Psi_{p}(u, y),
\end{aligned}
$$

and a similar expression can be written for $\partial^{2} \Psi_{p}(u, y) / \partial x \partial y$. In Eq. (16) the primes in $\varepsilon_{r}^{\prime}, \mu_{r}^{\prime}$, and $h_{r-1, r}^{\prime}$ denote derivatives with respect to $x$.

\section{Iterative Solutions to the Generalized Telegraphists' Equations for Rough Surface Scattering of the Radiation Fields}

Iterative analytical procedures as well as numerical techniques are used to solve the generalized telegraphists' equations [Eqs. (13) and (14)] for the forward and backward wave amplitude scattered by 2 -D rough surfaces. The analytical procedures are dealt with in this section. To obtain the single scatter approximations for the wave amplitudes, the expressions for the primary fields impressed upon the rough interface, due to the source, are first derived from Eqs. (13) and (14) on neglecting all the coupling terms manifested by the scattering coefficients $S_{P Q}^{B A}$. When the sources are in the far field, the primary, incident fields impressed on the rough surface are vertically and horizontally polarized plane waves propating in the direction of the (free space) wave vector $\overline{\mathbf{k}}_{o}^{i}=\mathbf{k}_{o x}^{i} \bar{a}_{x}+k_{o y}^{i} \bar{a}_{y}+k_{o z}^{i} \bar{a}_{z}=k_{o} \bar{n}^{i}$, where $\bar{n}^{i}$ is a unit vector and $k_{o}=\omega\left(\mu_{o} \varepsilon_{o}\right)^{1 / 2}$. Thus, the primary electric fields impressed upon the rough surfaces are

$$
\begin{aligned}
\overline{\mathbf{E}}^{i}= & \bar{a}^{P} E_{o}^{i} \exp \left[-i\left(k_{o x}^{i} x+k_{o z}^{i} z\right)\right] \\
& \times\left\{\exp \left(i k_{o y}^{i} y\right)+R_{o}^{P} \exp \left[i k_{o y}^{i}(2 h-y)\right]\right\},
\end{aligned}
$$

in which $R_{o}^{P}$ is the $P=V, H$ polarized Fresnel reflection coefficient for waves incident from medium 0 (free space) on medium 1 , and $\bar{a}^{P}$ is the unit vector in ( $P=V$, vertical/ parallel) or perpendicular $(P=H$, horizontal) to the plane of incidence. The primary fields are proportional to the lo- 
cal basis functions. The corresponding vertically or horizontally polarized field transforms and waves amplitudes are obtained using Eqs. (9), (10), and (12). In view of the biorthogonality relationships of Eq. (11), the primary wave amplitudes are proportional to the delta functions corresponding to the polarization $(Q=V, H)$ and direction $\bar{k}_{o}^{i}\left(u_{o}^{i}, v_{o}^{i} w_{o}^{i}\right)$ of the incident waves. When these expressions for the primary wave amplitudes are substituted for $a^{Q}$ and $b^{Q}$ on the right-hand sides of Eqs. (12) and (13) (with the source terms $A^{P}$ and $B^{P}$ suppressed) the (iterative) differential equations for single scattered wave amplitudes are obtained.

For plane wave excitations, the full-wave solution for the diffusely scattered propagating and evanescent waves from 2-D rough surfaces are expressed in matrix form as follows:

$$
\begin{aligned}
\mathbf{G}_{s}= & \left(\frac{k}{2 \pi i}\right)^{2} \int \frac{D\left(\overline{\mathbf{k}}^{\prime}, \overline{\mathbf{k}}^{i}\right)}{v_{y}^{\prime}}\left[\exp \left(-i \overline{\mathbf{v}}^{\prime} \cdot \overline{\mathbf{r}}_{s}\right)-\exp \left(i \overline{\mathbf{v}}^{\prime} \cdot \overline{\mathbf{r}}_{t}\right)\right] \\
& \times \frac{\mathrm{d} x_{s} \mathrm{~d} z_{s} \mathrm{~d} k_{y}^{\prime} \mathrm{d} k_{z}^{\prime}}{k_{x}^{\prime}} \mathbf{G}_{i},
\end{aligned}
$$

in which $\mathbf{G}_{s}$ and $\mathbf{G}_{i}$ are $2 \times 1$ column matrices whose elements are the vertically and horizontally polarized complex wave amplitudes for the scattered and incident waves, respectively. The elements of $2 \times 2$ matrix $\mathbf{D}\left(\bar{k}^{\prime}, \bar{k}^{i}\right)$ are the surface element scattering coefficients $\mathbf{D}^{P Q}\left(\bar{k}^{\prime}, \bar{k}^{i}\right)$ in which $P$ and $Q$ denote $V$ (vertical) or $H$ (horizontal) polarizations. They are given by

$\mathbf{D}\left(\bar{k}^{\prime}, \bar{k}^{\prime}\right)=2 \cos \theta^{\prime} \cos \theta^{i} R\left(\bar{k}^{\prime}, \bar{k}^{i}\right)$.

The wave vectors for the scattered and incident waves are respectively

$$
\begin{aligned}
\overline{\mathbf{k}}^{\prime} & =k\left(\sin \theta^{\prime} \cos \phi^{\prime} \bar{a}_{y}+\cos \theta^{\prime} \bar{a}_{y}+\sin \theta^{\prime} \sin \phi^{\prime} \bar{a}_{z}\right) \\
& =k_{x}^{\prime} \bar{a}_{x}+k_{y}^{\prime} \bar{a}_{y}+k_{z}^{\prime} \bar{a}_{z}
\end{aligned}
$$

and

$$
\begin{aligned}
\overline{\mathbf{k}}^{i} & =k\left(\sin \theta^{i} \cos \phi^{i} \bar{a}_{x}-\cos \theta^{i} \bar{a}_{y}+\sin \theta^{i} \bar{a}_{z}\right) \\
& =k_{x}^{i} \bar{a}_{x}+k_{y}^{i} \bar{a}_{y}+k_{z}^{i} \bar{a}_{z},
\end{aligned}
$$

in which $k=\omega(\mu \varepsilon)^{1 / 2}$ is the wave number in the medium above the rough interface (free space) and the incident wave impinges upon the medium with wave number $k_{1}$ $=\omega\left(\mu_{1} \varepsilon_{1}\right)^{1 / 2}$. The complex electric permittivity and magnetic permeability are $\varepsilon$ and $\mu$, respectively, and $\omega$ is the frequency in radians per second. The mean reference surface is given by $y=0$. The position vectors from the origin to the observation point and to points on the mean surface and to the rough surface respectively are

$\overline{\mathbf{r}}=x \bar{a}_{x}+y \bar{a}_{y}+z \bar{a}_{z}, \quad \overline{\mathbf{r}}_{t}=x_{s} \bar{a}_{x}+z_{s} \bar{a}_{z}$,

$\overline{\mathbf{r}}_{s}=\overline{\mathbf{r}}_{t}+h\left(x_{s}, z_{s}\right) \bar{a}_{y}$, in which $\bar{a}_{x}, \bar{a}_{y}$, and $\bar{a}_{z}$ are unit vectors.

The expressions for $\mathbf{R}^{P Q}\left(\overline{\mathbf{k}}^{\prime}, \overline{\mathbf{k}}^{i}\right)$ the elements of the matrix $\mathbf{R}\left(\overline{\mathbf{k}}^{\prime}, \overline{\mathbf{k}}^{i}\right)$ depend on the polarizations of the incident waves $Q=V, H$ and scattered waves $P=V, H$, the medium parameters on both sides of the interface, and the incident and scatter (spherical coordinate) angles $\theta^{i}, \phi^{i}$ and $\theta^{\prime}, \phi^{\prime}$ respectively. For flat surfaces $\mathbf{R}^{P P}$ are the Fresnel reflection coefficients and $\mathbf{R}^{P Q}=0$ for $P \neq Q$. In general the elements of the scattering matrix $\mathbf{R}\left(\overline{\mathbf{k}}^{\prime}, \overline{\mathbf{k}}^{i}\right)$ are, ${ }^{11}$

$\mathbf{R}^{V V}=\frac{\left[\mu_{r} C_{1}^{\prime} C_{1}^{i} \cos \left(\phi^{\prime}-\phi^{i}\right)-S_{0}^{\prime} S_{0}^{i}\right]\left(1-1 / \varepsilon_{r}\right)+\left(1-\mu_{r}\right) \cos \left(\phi^{\prime}-\phi^{i}\right)}{\left(C_{0}^{\prime}+\eta_{r} C_{1}^{\prime}\right)\left(C_{0}^{i}+\eta_{r} C_{1}^{i}\right)}$,

$\mathbf{R}^{H H}=\frac{\left[\varepsilon_{r} C_{1}^{\prime} C_{1}^{i} \cos \left(\phi^{\prime}-\phi^{i}\right)-S_{0}^{\prime} S_{0}^{i}\right]\left(1-1 / \mu_{r}\right)+\left(1-\varepsilon_{r}\right) \cos \left(\phi^{\prime}-\phi^{i}\right)}{\left(C_{0}^{\prime}+C_{1}^{\prime} / \eta_{r}\right)\left(C_{0}^{i}+C_{1}^{i} / \eta_{r}\right)}$,

$\mathbf{R}^{H V}=\frac{-\sin \left(\phi^{\prime}-\phi^{i}\right) n_{r}\left[\left(1-1 / \mu_{r}\right) C_{1}^{\prime}-\left(1-\varepsilon_{r}\right) C_{1}^{i}\right]}{\left(C_{0}^{\prime}+C_{1}^{\prime} / \eta_{r}\right)\left(C_{0}^{i}+\eta_{r} C_{1}^{i}\right)}$,

$\mathbf{R}^{V H}=\frac{\sin \left(\phi^{\prime}-\phi^{i}\right) n_{r}\left[\left(1-1 / \varepsilon_{r}\right) C_{1}^{\prime}-\left(1-\mu_{r}\right) C_{1}^{i}\right]}{\left(C_{0}^{\prime}+\eta_{r} C_{1}^{\prime}\right)\left(C_{0}^{i}+C_{1}^{i} / \eta_{r}\right)}$,

in which $C_{0}^{i}=\cos \theta_{0}^{i}, C_{0}^{\prime}=\cos \theta_{0}^{\prime}, S_{0}^{i}=\sin \theta_{0}^{i}, S_{0}^{\prime}=\sin \theta_{0}^{\prime}$. The corresponding quantities associated with medium 1 and denoted by the subscript 1 and angle $\theta_{1}$, are related to $\theta_{0}$ by Snell's law. The vector $\bar{v}^{\prime}$ is

$\overline{\mathbf{v}}^{\prime}=\overline{\mathbf{k}}^{\prime}-\overline{\mathbf{k}}^{i}=v_{x}^{\prime} \bar{a}_{x}+v_{y}^{\prime} \bar{a}_{y}+v_{z}^{\prime} \bar{a}_{z}$.

In Eq. (18) the integrations are over the rough surface (transverse) variables $x_{s}\left(-L_{x}, L_{x}\right)$ and $z_{s}\left(-L_{z}, L_{z}\right)$ as well as the wave vector variables $k_{y}^{\prime}$ and $k_{z}^{\prime}(-\infty, \infty)$. The first term $\mathbf{G}^{f}$ contains the $\operatorname{exponent} \exp \left(i v_{y} h\right)$, while the second term $\mathbf{G}_{d}^{f}$ does not. On integrating the second term with respect to $x_{s}$ and $z_{s}$, the delta functions are obtained

$\delta\left(k_{o x}^{\prime}-k_{o x}^{i}\right)=\frac{1}{2 \pi} \int_{-\infty}^{\infty} \exp \left(i v_{x}^{\prime} x_{s}\right) \mathrm{d} x_{s}$,

$\delta\left(k_{o z}^{\prime}-k_{o x}^{i}\right)=\frac{1}{2 \pi} \int_{-\infty}^{\infty} \exp \left(i v_{z}^{\prime} z_{s}\right) \mathrm{d} z_{s}$.

It is readily shown that the full-wave solution reduces to the small height-small slope perturbation solution of Rice $^{12}$ provided that it assumed that $k_{0} h \ll 1$. On retaining the first two terms of the Taylor series expansion of $\exp \left(i v_{y} h\right)$ it follows that

$\exp \left(i \overline{\mathbf{v}}^{\prime} \cdot \overline{\mathbf{r}}_{s}\right)-\exp \left(i \overline{\mathbf{v}}^{\prime} \cdot \overline{\mathbf{r}}_{t}\right) \approx i v_{y}^{\prime} h(x, z) \exp \left(i \overline{\mathbf{v}}^{\prime} \cdot \overline{\mathbf{r}}_{t}\right)$.

In this small height-small slope limit, the original fullwave solution, Eq. (18) is indistinguishable from the small perturbation solution for the far fields scattered by slightly rough surfaces. These limiting perturbation forms of the full-wave solutions are, however, no longer invariant to co- 


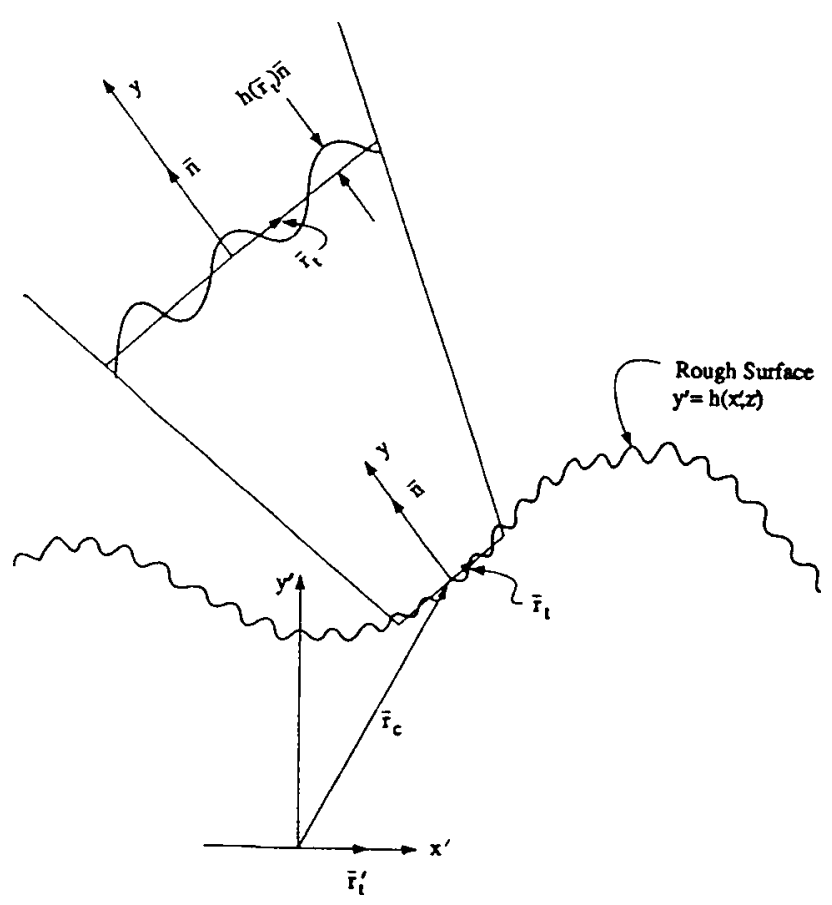

Fig. 1 Arbitrarily oriented patch on a rough surface. The fixed, reference coordinate system and the local, patch coordinate system.

ordinate transformations. Furthermore, it is shown that they are valid only if the height and slopes are of the same order of smallness.

Turing to the high-frequency limit, it is assumed that the radii of curvature of the larger scale rough surfaces are very large compared to wavelength. The unit vector $\bar{n}$ normal of these larger scale patches of rough surface is assumed to have arbitrary orientation (see Fig. 1). Thus the planes of incidence and scatter with respect to the reference coordinate systems (normal to $\bar{n}^{i} \times \bar{a}_{y}$ and $\bar{n}^{f} \times \bar{a}_{y}$, respectively) are not the same as the local planes of incidence and scatter with respect to the local coordinate systems (normal to $\bar{n}^{i}$ $\times \bar{n}$ and $\bar{n}^{f} \times \bar{n}$, respectively). Furthermore the sines and cosines of the angles of incidence and scatter appearing in the scattering coefficients [Eqs. (22) to (25)] are not the same as the sines and cosines of the local angles of incidence and scatter. To account for the arbitrary slope of the larger scale surface, the surface element scattering matrix in Eq. (19) is replaced by ${ }^{13}$

$\mathbf{D}\left(\overline{\mathbf{k}}^{f}, \overline{\mathbf{k}}^{i}\right) \rightarrow \mathbf{T}^{f} \mathbf{D}\left(\overline{\mathbf{k}}^{f}, \overline{\mathbf{k}}^{i}\right) \mathbf{T}^{i}$

In Eq. (30) the matrix operator $\mathbf{T}^{i}$ decomposes the waves that are vertically and horizontally polarized with respect to the reference plane of incidence (normal to $\bar{n}^{i} \times \bar{a}_{y}$ ) into vertically and horizontally polarized waves with respect to the local plane of incidence (normal to $\bar{n}^{i} \times \bar{n}$ ). Similarly, the matrix operator $\mathbf{T}^{f}$ decomposes the waves that are vertically and horizontally polarized with respect to the local plane of scatter (normal to $\bar{n}^{f} \times \bar{n}$ ) back into vertically and horizontally polarized waves with respect to the reference plane of scatter (normal to $\bar{n}^{f} \times \bar{a}_{y}$ ).

The changes represented by Eq. (30) constitute the transformation into the larger scale (patch) coordinate system (see Fig. 1). It is readily shown that at high frequencies, the major contributions come from the vicinity of the stationary phase, specular points on the rough surface where $\bar{n}$ is along the bisector between $\bar{n}^{f}$ and $-\bar{n}^{i}$. Pursuant to the transformation of Eq. (30), it can be shown that at these stationary phase points $\mathbf{R}^{V V}$ and $\mathbf{R}^{H H}$ reduce to the familiar Fresnel reflection coefficients while the cross polarized terms $\mathbf{R}^{V H}$ and $\mathbf{R}^{H V}$ vanish at the specular points. Thus in these limits, the full wave solution reduces to the physical optics solution for the diffuse scattered fields. ${ }^{14}$ If in addition Eq. (18) is evaluated analytically using stationary phase approximations, the full-wave solution reduces to the geometric optics solution. However, to account for multiple scatter at the same rough surface, it is necessary to return to the original form [Eq. (18)] even at high frequencies. ${ }^{15}$ Moreover, if the scales of roughness or medium fluctuations are smaller than a wavelength and near fields must be evaluated, the original form of the full-wave solution [Eq. (18)] must be used.

The mean intensities of the diffusely scattered like and cross polarized fields are

$$
\begin{aligned}
\left\langle\mathbf{G}_{S}^{P} \mathbf{G}_{S}^{P *}\right\rangle= & \left(\frac{k}{2 \pi}\right)^{4}\left\langle\int \frac{\mathbf{D}^{P Q}\left(\overline{\mathbf{k}}^{\prime}, \overline{\mathbf{k}}^{i}\right) \mathbf{D}^{P Q *}\left(\overline{\mathbf{k}}^{\prime \prime}, \overline{\mathbf{k}}^{i}\right)}{v_{y}^{\prime} v_{y}^{\prime \prime}}\right. \\
& \times \exp \left[-i\left(\overline{\mathbf{k}}^{\prime}-\overline{\mathbf{k}}^{\prime \prime}\right) \cdot \overline{\mathbf{r}}\right] \exp \left(i \overline{\mathbf{v}}^{\prime} \cdot \overline{\mathbf{r}}_{t}^{\prime}\right) \\
& \times\left[\exp \left(i v_{y}^{\prime} h^{\prime}\right)-1\right] \exp \left(-i \overline{\mathbf{v}}^{\prime \prime} \cdot \overline{\mathbf{r}}_{t}^{\prime \prime}\right) \\
& \times\left[\exp \left(-i v_{y}^{\prime \prime} h^{\prime \prime}\right)-1\right] \\
& \left.\times \frac{\mathrm{d} x_{s}^{\prime} \mathrm{d} z_{s}^{\prime} \mathrm{d} k_{y}^{\prime} \mathrm{d} k_{z}^{\prime} \mathrm{d} x_{s}^{\prime \prime} \mathrm{d} z_{s}^{\prime \prime} \mathrm{d} k_{y}^{\prime \prime} \mathrm{d} k_{z}^{\prime \prime}}{k_{x}^{\prime} k_{x}^{\prime \prime}}\right\rangle .
\end{aligned}
$$

The symbol $\langle\cdot\rangle$ denotes the statistical average. To facilitate the integration, the following changes of variables are made

$\overline{\mathbf{r}}_{t}^{\prime}-\overline{\mathbf{r}}_{t}^{\prime \prime}=\overline{\mathbf{r}}_{d},\left(\overline{\mathbf{r}}_{t}^{\prime}+\overline{\mathbf{r}}_{t}^{\prime \prime}\right) / 2=\overline{\mathbf{r}}_{c}$

Thus Eq. (31) is expressed as follows:

$$
\begin{aligned}
\left\langle\mathbf{G}_{S}^{P} \mathbf{G}_{S}^{P *}\right\rangle= & \left(\frac{k}{2 \pi}\right)^{4}\left\langle\int \frac{\mathbf{D}^{P Q}\left(\overline{\mathbf{k}}^{\prime}, \overline{\mathbf{k}}^{i}\right)}{v_{y}^{\prime}} \frac{\mathbf{D}^{P Q *}\left(\overline{\mathbf{k}}^{\prime \prime}, \overline{\mathbf{k}}^{i}\right)}{v_{y}^{\prime \prime}}\right. \\
& \times \exp \left[-i\left(\overline{\mathbf{k}}^{\prime}-\overline{\mathbf{k}}^{\prime \prime}\right) \cdot \overline{\mathbf{r}}\right] \exp \left[i\left(\overline{\mathbf{v}}^{\prime}+\overline{\mathbf{v}}^{\prime \prime}\right) \overline{\mathbf{r}}_{d} / 2\right] \\
& \times \exp \left[i\left(\overline{\mathbf{v}}^{\prime}-\overline{\mathbf{v}}^{\prime \prime}\right) \cdot \overline{\mathbf{r}}_{c}\right]\left[\exp \left(i v_{y}^{\prime} h^{\prime}\right)-1\right] \\
& \left.\times\left[\exp \left(-i v_{y}^{\prime \prime} h^{\prime \prime}\right)-1\right] \mathrm{d} \bar{r}_{c} \mathrm{~d} \bar{r}_{d} \frac{\mathrm{d} k_{y}^{\prime} \mathrm{d} k_{z}^{\prime} \mathrm{d} k_{y}^{\prime \prime} \mathrm{d} k_{z}^{\prime \prime}}{k_{x}^{\prime} k_{x}^{\prime \prime}}\right\rangle .
\end{aligned}
$$

For points on the surface, the significant contributions to the fields come from the small Fresnel zone ${ }^{16}$ around the points at $\overline{\mathbf{r}}_{t}^{\prime}$ and $\overline{\mathbf{r}}_{t}^{\prime \prime}$. On integrating Eq. (25) with respect to $\overline{\mathbf{r}}_{c}$, therefore, the limits of integration can be assumed to be infinite. Furthermore, 


$$
\begin{gathered}
\frac{1}{(2 \pi)^{2}} \int_{-\infty}^{\infty} \exp \left[i\left(\overline{\mathbf{v}}^{\prime}-\overline{\mathbf{v}}^{\prime \prime}\right) \cdot \overline{\mathbf{r}}_{c}\right] \mathrm{d} x_{c} \mathrm{~d} z_{c} \\
=\delta\left(v_{x}^{\prime}-v_{x}^{\prime \prime}\right) \delta\left(v_{z}^{\prime}-v_{z}^{\prime \prime}\right)
\end{gathered}
$$

Thus integrating the results with respect to $\mathrm{d} k_{y}^{\prime \prime} \mathrm{d} k_{z}^{\prime \prime}$ and noting that $\mathrm{d} k_{y} / k_{x}=-\mathrm{d} k_{x} / k_{y}$, Eq. (25) reduces to

$$
\begin{aligned}
\left\langle\mathbf{G}_{S}^{P} \mathbf{G}_{S}^{P *}\right\rangle= & \frac{k^{4}}{(2 \pi)^{2}}\left\langle\int\left|\frac{\mathbf{D}^{P Q}\left(\overline{\mathbf{k}}^{\prime}, \overline{\mathbf{k}}^{i}\right)}{v_{y}^{\prime}}\right|^{2}\right. \\
& \times \exp \left(i \overline{\mathbf{v}}^{\prime} \cdot \overline{\mathbf{r}}_{d}\right)\left[\exp \left(i v_{y}^{\prime} h^{\prime}\right)-1\right] \\
& \left.\times\left[\exp \left(-i v_{y}^{\prime} h^{\prime \prime}\right)-1\right] \mathrm{d} \overline{\mathbf{r}}_{d} \frac{\mathrm{d} k_{x}^{\prime} \mathrm{d} k_{z}^{\prime}}{\left(k_{y}^{\prime}\right)^{2}}\right\rangle .
\end{aligned}
$$

For nanostructures with scales of roughness much smaller than the wavelength of the illuminating source, $k h \ll 1$. We approximate $\left[\exp \left(i v_{y} h\right)-1\right]$ with $\left(i v_{y} h\right)$, etc., and note that the Fourier transform of the surface height autocorrelation function $\left\langle h^{\prime} h^{\prime \prime}\right\rangle$ is the surface height spectral density function $W$.

$\frac{1}{(2 \pi)^{2}} \int_{-\infty}^{\infty}\left\langle h^{\prime} h^{\prime \prime}\right\rangle \exp \left(i \overline{\mathbf{v}}^{\prime} \cdot \overline{\mathbf{r}}_{d}\right) \mathrm{d} \overline{\mathbf{r}}_{d}=W\left(v_{x}^{\prime}, v_{z}^{\prime}\right)$.

Thus Eq. (27) reduces to

$\left\langle\mathbf{G}_{S}^{P} \mathbf{G}_{S}^{P *}\right\rangle=k^{4} \int\left|\frac{\mathbf{D}^{P Q}\left(\overline{\mathbf{k}}^{\prime}, \overline{\mathbf{k}}^{i}\right)}{k_{y}^{\prime}}\right|^{2} W\left(v_{x}^{\prime}, v_{z}^{\prime}\right) \mathrm{d} k_{x}^{\prime} \mathrm{d} k_{z}^{\prime}$.

Note that the result in Eq. (29) accounts for both propagating waves, with $k_{y}=\left(1-k_{x}^{2}-k_{z}^{2}\right)^{1 / 2}$ real and evanescent waves, with $k_{y}$ imaginary. Both must be accounted for when fields very near the surface are evaluated.

Since the surface height spectral density function $W\left(v_{x}^{\prime}, v_{z}^{\prime}\right)$ (related to the surface height autocorrelation function) appears in the expression for the near fields scattered by nanoscale surface roughness, this work can be applied to the synthesis and modification of nanostructure surfaces.

It is interesting to compare the above results with those for the high-frequency limit with $k h>>1$. For this case, the following substitutions are made in Eq. (26):

$h^{\prime}=h_{c}+\left(x_{d} h_{x}+z_{d} h_{z}\right) / 2$ and $h^{\prime \prime}=h_{c}-\left(x_{d} h_{x}+z_{d} h_{z}\right) / 2$,

where

$h_{c}=\left(h^{\prime}+h^{\prime \prime}\right) / 2, \quad h_{x}=\frac{\partial h}{\partial x}$, and $h_{z}=\frac{\partial h}{\partial z}$.

Thus for the high frequency limit,

$$
\begin{aligned}
\left\langle\mathbf{G}_{S}^{P} \mathbf{G}_{S}^{P *}\right\rangle= & \frac{k^{4}}{(2 \pi)^{2}}\left\langle\int\left|\frac{\mathbf{D}^{P Q}\left(\overline{\mathbf{k}}^{\prime}, \overline{\mathbf{k}}^{i}\right)}{v_{y}^{\prime}}\right|^{2}\right. \\
& \times \exp \left\{i\left[v_{x}^{\prime} x_{d}+v_{y}^{\prime}\left(x_{d} h_{x}+z_{d} h_{z}\right)+v_{z}^{\prime} z_{d}\right]\right\} \\
& \left.\times \mathrm{d} x_{d} \mathrm{~d} z_{d} \frac{\mathrm{d} k_{x}^{\prime} \mathrm{d} k_{z}^{\prime}}{\left(k_{y}^{\prime}\right)^{2}}\right\rangle \\
= & k^{4}\left\langle\int\left|\frac{\mathbf{D}^{P Q}\left(\overline{\mathbf{k}}^{\prime}, \overline{\mathbf{k}}^{i}\right)}{v_{y}^{\prime}}\right|^{2} \delta\left(v_{x}^{\prime}+v_{y}^{\prime} h_{x}\right)\right. \\
& \left.\times \delta\left(v_{z}^{\prime}+v_{y}^{\prime} h_{z}\right) \frac{\mathrm{d} k_{x}^{\prime} \mathrm{d} k_{z}^{\prime}}{\left(k_{y}^{\prime}\right)^{2}}\right\rangle \\
= & k^{4} \int\left|\mathbf{D}^{P Q}\left(\overline{\mathbf{k}}^{\prime}, \overline{\mathbf{k}}^{i}\right)\right|_{S}^{2} p\left(h_{x s}, h_{z s}\right) \frac{\mathrm{d} k_{x}^{\prime} \mathrm{d} k_{z}^{\prime}}{\left(v_{y}^{\prime}\right)^{4}\left(k_{y}^{\prime}\right)^{2}},
\end{aligned}
$$

in which $\mathbf{D}^{P Q}$ and the slope probability density function $p\left(h_{x}, h_{z}\right)$ are evaluated at the specular points where

$h_{x s}=-\frac{v_{x}^{\prime}}{v_{y}^{\prime}}=-\left(\frac{k_{x}^{\prime}-k_{x}^{i}}{k_{y}^{\prime}-k_{y}^{i}}\right)$,

and

$h_{z s}=-\frac{v_{z}^{\prime}}{v_{y}^{\prime}}=-\left(\frac{k_{z}^{\prime}-k_{z}^{i}}{k_{y}^{\prime}-k_{y}^{i}}\right)$,

and the unit vector normal to the surface at the specular points are

$\bar{n}_{s}=\left(-h_{x s} \bar{a}_{x}+\bar{a}_{y}-h_{z s} \bar{a}_{z}\right) /\left(1+h_{x s}^{2}+h_{z s}^{2}\right)^{1 / 2}$.

Since the surface slope probability density function appears in the expression for the near fields scattered by larger scale roughness (with nonnegligible slopes), this work can be extended to the synthesis and modification of nanostructure surfaces with undulating slopes. The expression for the scattered power density (average Poynting vectors) can be evaluated in manner similar to Eq. (23). The ratio between the horizontal components of the electric and magnetic fields is given by the wave impedances $\omega \mu / k_{y}$ and $k_{y} / \omega \varepsilon$ for horizontally and vertically polarized waves, respectively. For evanescent waves, $k_{y}$ is imaginary, thus the horizontal components of the electric and magnetic fields are $\pi / 2$ radians out of phase, corresponding to no average scattered power flow in the vertical direction associated with evanescent waves.

The full-wave analysis can be readily applied to nearand/or far-field scattering from high-density quantum dot arrays used in nonlinear optics, data storage, and other electronic applications. These structures are usually (quasi) periodic. For 2-D periodic structures, the full-wave solutions for the scattered fields corresponding to Eq. (18) is 


$$
\begin{aligned}
\mathbf{G}_{S}= & \left(\frac{k}{2 \pi i}\right)^{2} \int A_{x}\left(N_{x} v_{x}^{\prime} L_{x}\right) A_{z}\left(N_{z} v_{z}^{\prime} L_{z}\right) \frac{\mathrm{D}\left(\overline{\mathbf{k}}^{\prime}, \overline{\mathbf{k}}^{i}\right)}{v_{y}^{\prime}} \\
& \times \exp \left(-i \overline{\mathbf{k}}^{\prime} \cdot \overline{\mathbf{r}}\right)\left[\exp \left(i \overline{\mathbf{v}}^{\prime} \cdot \overline{\mathbf{r}}_{s}\right)-\exp \left(i \overline{\mathbf{v}}^{\prime} \cdot \overline{\mathbf{r}}_{t}\right)\right. \\
& \times \frac{\mathrm{d} x_{s} \mathrm{~d} z_{s} \mathrm{~d} k_{y}^{\prime} \mathrm{d} k_{z}^{\prime}}{k_{x^{\prime}}} \mathrm{G}_{i},
\end{aligned}
$$

in which the $\left(N_{x} \cdot N_{z}\right)$ element array factors are

$$
\begin{aligned}
& A_{x}\left(N_{x} v_{x}^{\prime} L_{x}\right)=\frac{\sin \left(N_{x} v_{x}^{\prime} L_{x} / 2\right)}{\sin \left(v_{x}^{\prime} L_{x} / 2\right)} \exp \left(-i v_{x}^{\prime} L_{x} / 2\right), \\
& A_{z}\left(N_{z} v_{z}^{\prime} L_{z} / 2\right)=\frac{\sin \left(N_{z} v_{z}^{\prime} L_{z} / 2\right)}{\sin \left(v_{z}^{\prime} L_{z} / 2\right)} \exp \left(-i v_{z}^{\prime} L_{z} / 2\right) .
\end{aligned}
$$

The integrations with respect to $\mathrm{d} x_{s} \mathrm{~d} z_{s}$ are over the area $L_{x} L_{z}$ of one element of the periodic structure. The integrations with respect to $\mathrm{d} k_{y}^{\prime} \mathrm{d} k_{z}^{\prime}$ are as in Eq. (18), over the propagating and evanescent spectra of a scattered waves. A small random rough component can also be added to the (quasi) periodic surface height. When the quasiperiodic surface height has a small random component $h=h_{p}+h_{r}$ (subscripts $p$ and $r$ denote periodic and random, respectively) the statistical average (over random heights) of Eq. (41) is first obtained, involving the surface height characterization functions.

For observation points near the surface, the full-wave solutions account for coupling between the propagating and evanescent waves, the lateral waves and the surface waves associated with rough surface scattering. When the rough surface is assumed to be perfectly conducting, the contribution from the branch-cut integral associated with the lateral waves vanishes and there are no residue contributions (associated with surface waves) from the singularities of the reflection coefficients. When the approximate impedance boundary condition is used, the lateral wave contribution is eliminated. Since the perturbation and physical optics solutions are far-field approximations, the surface wave and the lateral waves are not accounted for in these solutions.

The full-wave method can also be used to determine the fields scattered on transmission across rough surfaces. ${ }^{17}$ When scattering from more than one rough interface in irregular stratified media is considered, in general, it becomes necessary to account for scattering upon reflection and transmission across rough interfaces.

\section{Coupling between the Radiation Fields, the Lateral Waves, and the Surface Waves}

For simplicity, in this section, the rough surface height and the medium parameters are assumed to be independent of the $z$ variable and the incident wave vector $\overline{\mathbf{k}}^{i}$ is restricted to the $x y$ plane. Thus, the scattered wave vectors $\overline{\mathbf{k}}^{\prime}$ are also restricted to the $x, y$ plane and the scattered waves are not depolarized. Thus, the uppercase superscripts or subscripts $P, Q=V, H$ are dropped from the notation for the generalized telegraphists' equation. Instead the low case subscripts $p$ and $q$ are inserted to identify the particular contribution to the complete wave spetrum. Thus $p, q=0$ identifies the radiation terms; $p, q=1$ identifies the lateral wave term (associated with the branch cuts that end on the branch points at $k_{o}$ and $k_{m}$, the wave numbers for the uppermost and lowermost media) and $p, q=s$ identifies the surface wave terms associated with the residues at the poles of the total reflection coefficients. The generalized telegraphists' equations are expressed as follows:

$$
\begin{aligned}
& \frac{-\mathrm{d} a_{p}}{\mathrm{~d} x}-i u a_{p}=\sum_{q} S_{p q}^{B A} a_{q}+S_{p q}^{B B} b_{q}-A^{P}, \\
& \frac{-\mathrm{d} b_{p}}{\mathrm{~d} x}+i u b_{p}=\sum_{q} S_{p q}^{A A} a_{q}+S_{p q}^{A B} b_{q}+B^{P} .
\end{aligned}
$$

Thus in Eqs. (43) and (44) the scattering coefficients $S_{10}^{\alpha \beta}$ and $S_{s 0}^{\alpha \beta}(\alpha, \beta=A, B)$ account for coupling between the incident radiation fields and the scattered lateral waves and surface waves respectively. Explicit expressions for these rough surface scattering coefficients and the reciprocal expressions $S_{01}^{\alpha \beta}$ and $S_{0 s}^{\alpha \beta}$ have been published ${ }^{18,19}$ for both vertically and horizontally polarized excitations. Iterative, single scatter solutions to Eqs. (43) and (44) are derived using the procedures used to derive the scattered radiation fields. Thus the primary, source dependent impressed fields are obtained from Eqs. (43) and (44) on neglecting the coupling terms. The first-order scattering terms are obtained on substituting the expressions for the impressed fields on the right-hand sides of Eqs. (43) and (44) and suppressing the source terms $A^{P}$ and $B^{P}$. The resulting expressions for the lateral wave or surface wave amplitudes are substituted into Eqs. (7) and (8) to obtain the electric and magnetic field expressions associated with the lateral waves and the surface waves that are excited by the radiation fields incident on the rough surface.

\section{Concluding Remarks}

A full-wave approach based on the complete expansions of the scattered electromagnetic fields into the radiation term, lateral waves, and surface waves associated with layered media have been used to convert Maxwell's equations into generalized telegraphists' equations. Exact boundary conditions are imposed at the rough interface between two laterally fluctuating media. The coupling between the wave amplitudes is due to surface height and medium fluctuations. Both random and periodic rough surfaces are considered in the analysis of the scattered near fields. Both large-scale and small-scale (including subwavelength) features of nanostructures can be characterized. This work is applicable to the synthesis of nanostructures to modify the mechanical and/or electrical characteristics. These include the enhancement of lubrication between surfaces, the avoidance of adhesion between surfaces, and the modification of the electromagnetic characteristics of quantum dots.

\section{References}

1. E. Bahar, "Depolarization of electromagnetic waves excited by distribution of electric and magnetic sources in inhomogeneous multilayered structures of arbitrarily varying thickness-generalized field transform," J. Math. Phys. 14(11), 1502-1509 (1973). 
2. E. Bahar, "Depolarization of electromagnetic waves excited by distribution of electric and magnetic sources in inhomogeneous multilayered structures of arbitrarily varying thickness-full wave solutions,' J. Math. Phys. 14(11), 1510-1515 (1973).

3. E. Bahar, "Depolarization in nonuniform multilayered structuresfull wave solutions," J. Math. Phys. 15(2), 202-208 (1974).

4. E. Bahar, "Generalized Fourier transform for stratified media," Can. J. Phys. 50(24), 3123-3131 (1972).

5. E. Bahar, "Radio wave propagation in stratified media with nonuniform boundaries and varying electromagnetic parameters-full wave analysis," Can. J. Phys. 50(24), 3132-3142 (1972).

6. S. A. Schelkunoff, "Conversion of Maxwell's equations into generalized telegraphists' equations," Bell Syst. Tech. J. 34, 995-1045 (1955).

7. E. Bahar and G. Govindarajan, "Radiation by a line source over nonuniform stratified earth," J. Geophys. Res. 78(2), 393-406 (1973).

8. E. Bahar, "Field transforms for multilayered cylindrical and spherical structures of finite conductivity," Can. J. Phys. 53(11), 1078-1087 (1975).

9. E. Bahar, "Propagation in irregular multilayaered cylindrical structures of finite conductivity-full wave solutions," Can. J. Phys. 53(11), 1088-1096 (1975).

10. E. Bahar, "Full wave solutions for the depolarization of the scattered radiation fields by rough surface of arbitrary slope," IEEE Trans. Antennas Propag. AP-29(3), 443-454 (1981).

11. E. Bahar and Y. Zhang, "A new unified full wave approach to evaluate the scatter cross sections of composite random rough surfaces," IEEE Trans. Geosci. Remote Sens. 34(4), 973-980 (1996).

12. S. O. Rice, "Reflection of electromagnetic waves from a slightly rough surface," Commun. Pure Appl. Math. 4, 351-378 (1951).

13. E. Bahar, C. L. Rufenach, P. E. Barrick, and M. A. Fitzwater, "Scattering cross section modulation for arbitrarily oriented composite rough surfaces-full wave solutions," Radio Sci. 18(5), 675-690 (1983).

14. E. Bahar and B. S. Lee, "Full wave solutions for rough surface bistatic radar cross sections: comparison with small pertrubation, physical optics, numerical and experimental results," Radio Sci. 29(2), 407-429 (1994)

15. E. Bahar and M. El-Shenawee, "Enhanced backscatter from one- dimensional random rough surfaces: stationary-phase approximations to full wave solutions," J. Opt. Soc. Am. A 12(1), 151-161 (1995).

16. P. Beckmann and A. Spizzichino, The Scattering of Electromagnetic Waves from Rough Surfaces, Macmillan, New York (1963).

17. E. Bahar and B. S. Lee, "Transmission scatter cross sections across two-dimensional random rough surfaces-full wave solutions and comparison with numerical results," Waves Random Media 6, 25-48 (1996).

18. E. Bahar, "Excitation of lateral waves and the scattered radiation fields by rough surfaces of arbitrary slope," Radio Sci. 15(6), 10951104 (1980).

19. E. Bahar, "Excitation of surface waves and the scattered radiation fields by rough surfaces of arbitrary slope," IEEE Trans. Microwave Theory Tech. MTT-28(9), 999-1006 (1980).

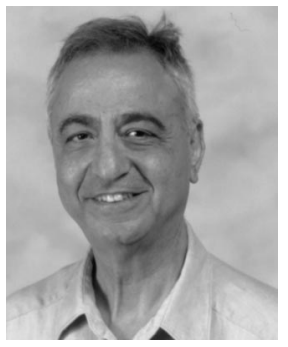

Ezekiel Bahar received his BS and MS degrees from the Technion-Israel Institute of Technology, Haifa, in 1958 and 1960, respectively, and his $\mathrm{PhD}$ degree from the University of Colorado, Boulder, in 1964. He joined the Department of Electrical Engineering, University of Nebraska-Lincoln, as an associate professor in 1967. In 1971, he became a professor of electrical engineering and he is currently a University Professor and the George Holmes Professor of electrical engineering. He designed laboratory models to investigate EM propagation in nonuniform terrestrial waveguides. $\mathrm{He}$ developed full-wave analytical and numerical solutions to problems of diffraction, scattering, and depolarization of radio waves in nonuniform layered structures with random rough interfaces and inhomogeneous, anisotropic, chiral materials. Dr. Bahar is a fellow of the IEEE and a member of Commissions B, C, F, and G of the International Union of Radio Science and IEEE Antennas and Propagation Society. 